\title{
Investigation of Room-Temperature Multiwavelength Fiber-Ring Laser That Incorporates an SOA-Based Phase Modulator in the Laser Cavity
}

\author{
Jian Yao, Jianping Yao, Senior Member, IEEE, Member OSA, Zhichao Deng, and Jian Liu
}

\begin{abstract}
A theoretical and experimental study of a multiwavelength fiber-ring laser that incorporates a semiconductor optical amplifier (SOA) in the laser cavity as a phase modulator to suppress the homogeneous line broadening is presented. The analysis reveals that the phase shift introduced by the SOA-based phase modulator is more significant than that formed using a $\mathrm{LiNbO}_{3}$ phase modulator, which leads to a better suppression of the homogeneous line broadening. Multiwavelength lasing with small wavelength spacing and improved stability at room temperature is achieved. A fiber-ring laser based on the proposed approach is implemented. Stable multiwavelength operation with up to 26 wavelengths and wavelength spacing as small as $0.19 \mathrm{~nm}$ at room temperature is demonstrated.
\end{abstract}

Index Terms-Erbium-doped fiber (EDF), fiber laser, homogeneous line broadening, multiwavelength, phase modulation, semiconductor optical amplifier (SOA).

\section{INTRODUCTION}

$\mathbf{F}$ IBER lasers that can generate stable multiwavelengths are of great interest for many applications such as wavelengthdivision multiplexing (WDM) communications, fiber-optic sensors, optical instrumentation, and microwave photonic systems. Some of the main challenges for erbium-doped fiber (EDF) lasers are the strong homogeneous line broadening and the cross-gain saturation that make it difficult to achieve stable multiwavelength lasing with small wavelength spacing at room temperature. Many approaches have been proposed recently [1]-[7]. One direct solution to this problem is to cool the EDF in liquid nitrogen $(77 \mathrm{~K})$ to suppress the homogeneous line broadening and the cross-gain saturation [1]-[3], but the technique is not suitable for practical applications because of its complexity and high cost. To achieve stable multiwavelength lasing, Graydon et al. [4] proposed to use a specially designed twin-core fiber; by doping both the cores with erbium, a gain medium with a large scale of gain inhomogeneity is achieved. A fiber laser incorporating the length of the erbium-doped twincore fiber as the gain medium with a stable multiwavelength lasing was demonstrated [4]. To control the homogeneous line

Manuscript received October 12, 2004; revised May 9, 2005. This work was supported by the National Capital Institute of Telecommunications and Natural Sciences and Engineering Research Council of Canada.

J. Yao, J. Yao, and Z. Deng are with the Microwave Photonics Research Laboratory, School of Information Technology and Engineering, University of Ottawa, Ottawa, ON K1N 6N5, Canada (e-mail: jpyao@ site.uottawa.ca).

$\mathrm{J}$. Liu is with the Institute for National Measurement Standards, National Research Council of Canada, Ottawa, ON K1A 0R6, Canada.

Digital Object Identifier 10.1109/JLT.2005.850818 broadening, Zhao et al. [5] proposed to use an optical feedback that functions through adjusting the dynamic inhomogeneous saturated gains at different lasing wavelengths. More recently, a multiwavelength fiber-ring laser operating at room temperature was reported by Bellemare et al. [6] in which stable multiwavelength operation was realized by incorporating an acoustooptic frequency shifter. A stable multiwavelength lasing was realized at room temperature. However, the acoustooptic frequency shifter used in the configuration has a high insertion loss. To solve this problem, Zhou et al. [7] proposed to use an electrooptic phase modulator or an all-fiber phase modulator driven by a piezoelectric transducer (PZT) to replace the acoustooptic frequency shifter. Multiwavelength lasing at room temperature was demonstrated when the phase modulator was driven by a sinusoidal signal. A major drawback of this technique is that the sinusoidal signal applied to the phase modulator requires a high drive voltage, and the tension frequently applied to the all-fiber phase modulator leads to a reduced lifetime.

We have recently demonstrated a fiber laser that can generate stable multiwavelength lasing at room temperature [8]. In the proposed laser configuration, a semiconductor optical amplifier (SOA) was incorporated in the laser cavity in which the SOA was biased just above the transparent point with a gain of about $2 \mathrm{~dB}$. The homogeneous line broadening and crossgain saturation of the EDF in the laser cavity were greatly suppressed. Stable multiwavelength lasing with up to eight wavelengths at room temperature was demonstrated. Very recently, we demonstrated a different multiwavelength fiber laser that can generate more wavelengths at room temperature with improved stability [9]. In the laser configuration, an SOA biased just below the transparent point, but modulated with a lowvoltage sinusoidal signal, is incorporated in the laser cavity. Better performance in terms of lasing stability and number of wavelength was achieved compared with the approach in [8]. In this paper, we will present a theoretical study of the multiwavelength fiber laser that was experimentally demonstrated in [9]. Theoretical analysis shows that the phase shift introduced by the SOA is more significant than that formed by a $\mathrm{LiNbO}_{3}$ electrooptic modulator, which provides an improved homogeneous line broadening suppression. Stable lasing with more wavelengths and smaller wavelength spacing at room temperature is possible. A fiber-ring laser based on the proposed configuration is implemented. Stable multiwavelength operation at room temperature with up to 26 wavelengths and a wavelength spacing as small as $0.19 \mathrm{~nm}$ is realized. 


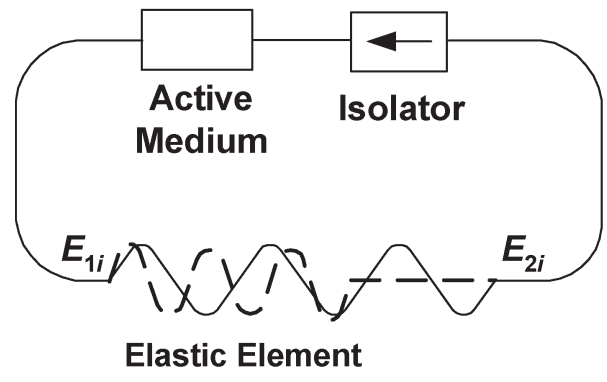

Fig. 1. A simplified fiber-ring laser model.

\section{PRINCIPLE OF OPERATION}

Based on stability analysis [10], it is known that any small perturbation in the laser cavity will result in the energy distribution change of the laser modes. In an EDF-based ring laser, different modes experience different net gains, depending on the polarization state and the wavelength. Because of homogeneous gain broadening of the EDF, the fiber laser suffers from a strong mode hopping, which makes it impossible to generate stable multiwavelength lasing at room temperature.

By solving the rate equation, one nontrivial stable solution can be obtained [11]. However, due to the change of energy distribution over the lasing wavelengths, which is resulted from homogeneous line broadening, the oscillation modes are competing to be dominant over others. When an SOA modulated at low frequency is incorporated into the ring laser cavity, it acts as an optical cavity buffer, producing elastic compression and expansion of the cavity length. Assuming that the phase modulation provided by the SOA is $\phi_{\mathrm{m}}(t)$ at a modulation frequency $\omega_{\mathrm{m}}$, the cavity length will be shifted by $\phi_{\mathrm{m}}(t) / \beta$ at the frequency $\omega_{\mathrm{m}}$, where $\beta$ is the propagation constant of light in the fiber. When the rate of the cavity length shift is comparable with the relaxation time of the laser, none of oscillation modes will be temporarily dominant over others; a simultaneous multiwavelength lasing is possible. A more detailed theoretical analysis is given below.

Fig. 1 shows a simplified ring cavity model in which an SOA acts as an elastic element to produce a phase shift of quantity $\phi_{\mathrm{m}}(t)$. The electrical field $E_{2 i}(t)$ of the $i$ th mode of the signal leaving the elastic element can be obtained by the electrical field $E_{1 i}(t)$ of the $i$ th mode arriving at the elastic element

$$
E_{2 i}(t)=E_{0 i} \mathrm{e}^{j\left[\omega_{0 i} t+\phi_{i}(t)\right]}=E_{1 i}(t) \mathrm{e}^{j \phi_{\mathrm{m}}(t)}
$$

where $E_{0 i}$ is the amplitude of the $i$ th mode. If the round-trip net gain is unity and the dispersion of the fiber and passive components are neglected, then $E_{1 i}(t) \approx E_{2 i}(t-T)$, where $T$ is the round-trip period of the signal. Thus

$$
E_{0 i} \mathrm{e}^{j\left[\omega_{0 i} t+\phi_{i}(t)\right]}=E_{0 i} \mathrm{e}^{j\left[\omega_{0 i}(t-T)+\phi_{i}(t-T)\right]} \mathrm{e}^{j \phi_{\mathrm{m}}(t)} .
$$

Since $\omega_{0 i} T=2 k \pi$, where $k$ is an integer, we could obtain

$$
\phi_{i}(t)=\phi_{i}(t-T)+\phi_{\mathrm{m}}(t) .
$$

The single-pass phase that resulted from the SOA is given by [12]

$$
\phi_{\mathrm{s}}(t)=\frac{2 \pi L}{\lambda}\left(n+\frac{\mathrm{d} n}{\mathrm{~d} N} \Delta N(t)\right)
$$

where $n$ is the refractive index of the active layer, $\mathrm{d} n / \mathrm{d} N$ is the refractive-index shift coefficient, $L$ is the length of the active region of the SOA, and $\Delta N(t)$ is the carrier density variation that is a function of the pump current change $\Delta I(t)$. It can be expressed by using an empirical value of $\mathrm{d} N / \mathrm{d} I=$ $8 \times 10^{16} \mathrm{~cm}^{-3} \mathrm{~mA}^{-1}[13]$

$$
\Delta N(t)=\frac{\mathrm{d} N}{\mathrm{~d} I} \Delta I(t)
$$

Therefore, the single-pass phase change with the external modulation signal on the SOA is

$$
\phi_{\mathrm{m}}(t)=\frac{2 \pi L}{\lambda} \frac{\mathrm{d} n}{\mathrm{~d} N} \frac{\mathrm{d} N}{\mathrm{~d} I} \frac{V(t)}{R}
$$

where $R$ is the impedance of the signal port of the SOA $(50 \Omega)$ and $V(t)$ represents the external modulation voltage. Let us consider that the modulator is driven by a sinusoidal signal

$$
V(t)=V_{0} \cos \left(\omega_{\mathrm{m}} t\right) .
$$

By solving (3), (6), and (7), the phase $\phi_{i}(t)$ of the oscillation signal in the ring laser will have a similar modulation

$$
\phi_{i}(t)=\frac{2 \pi L}{\lambda} \frac{\mathrm{d} n}{\mathrm{~d} N} \frac{\mathrm{d} N}{\mathrm{~d} I} \frac{1}{R} \frac{V_{0}}{2 \sin \left(\frac{\omega_{\mathrm{m}} T}{2}\right)} \sin \omega_{\mathrm{m}}\left(t+\frac{T}{2}\right) .
$$

The instantaneous frequency $\omega_{i}(t)=\mathrm{d} \phi_{\text {tot }}(t) / \mathrm{d} t$ in which $\phi_{\text {tot }}$ is the total phase of the signal can be obtained as

$$
\begin{aligned}
\omega_{i}(t) & =\frac{\mathrm{d}\left(\omega_{0 i} t+\phi_{i}(t)\right)}{\mathrm{d} t} \\
& =\omega_{0 i}+\frac{\pi L V_{0}}{\lambda R} \frac{\mathrm{d} n}{\mathrm{~d} N} \frac{\mathrm{d} N}{\mathrm{~d} I} \frac{\omega_{\mathrm{m}}}{\sin \left(\frac{\pi \omega_{\mathrm{m}}}{\Delta \omega_{\mathrm{a}}}\right)} \cos \omega_{\mathrm{m}}\left(t+\frac{T}{2}\right)
\end{aligned}
$$

where $\omega_{0 i}$ is the fundamental frequency and $\Delta \omega_{\mathrm{a}}$ is the axial mode spacing.

It can be seen that the SOA phase modulation produces a frequency modulation of the lasing signal, hence permitting a frequency shift in the laser cavity. When the SOA is modulated with a low-frequency signal ( $\sim$ in kilohertz) $\omega_{\mathrm{m}} \ll \Delta \omega_{\mathrm{a}}$, the frequency modulation that resulted from the SOA can be obtained as

$$
\omega_{i}(t) \approx \omega_{0 i}+\frac{L V_{0} \Delta \omega_{\mathrm{a}}}{\lambda R} \frac{\mathrm{d} n}{\mathrm{~d} N} \frac{\mathrm{d} N}{\mathrm{~d} I} \cos \omega_{\mathrm{m}}\left(t+\frac{T}{2}\right) .
$$

According to (10), for a given fiber laser, the amount of frequency shift is determined by the modulation voltage $V_{0}$ and the modulation frequency $\omega_{\mathrm{m}}$. Considering a fiber laser with a typical EDF length of $50 \mathrm{~m}$, if a $300-\mathrm{kHz}$ sinusoidal modulation 


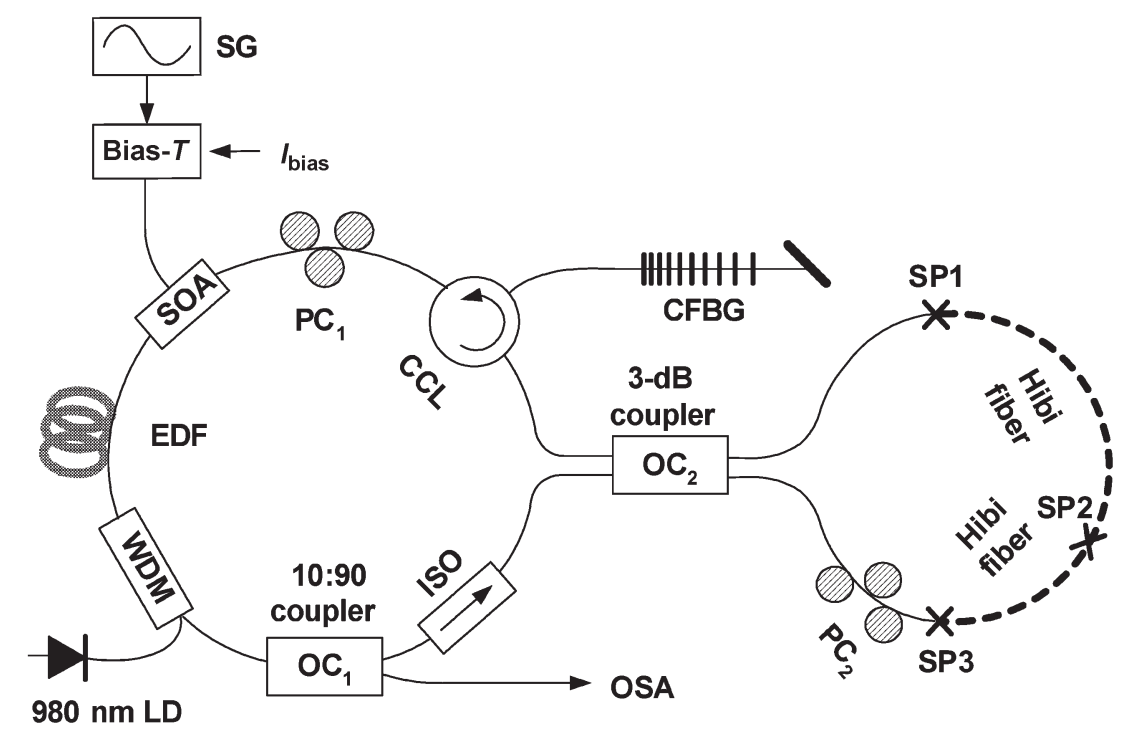

Fig. 2. Configuration of the multiwavelength EDF laser. WDM: 980-nm wavelength division multiplexer, OC: optical coupler, ISO: isolator, CCL: optical circulator, PC: polarization controller, SP: splicing point, CFBG: chirped fiber Bragg grating, SG: signal generator, OSA: optical spectrum analyzer.

signal is applied to the SOA, we have $\omega_{\mathrm{m}}[t+(T / 2)] \leq 0.7$, which is smaller than $\pi$. Therefore, $\cos \omega_{\mathrm{m}}[t+(T / 2)]$ is a monotonically decreasing function. To maintain the same frequency shift, it has to indicate that a higher modulation voltage is required for a higher modulation frequency.

Taking the typical values $\mathrm{d} n / \mathrm{d} N=-2 \times 10^{-20} \mathrm{~cm}^{3}, L=$ $500 \mu \mathrm{m}$, and $\mathrm{d} N / \mathrm{d} I=8 \times 10^{16} \mathrm{~cm}^{-3} \mathrm{~mA}^{-1}$ [13] from (6) and (7), the phase change at $1550 \mathrm{~nm}$ that resulted from the external modulation is approximated to be

$$
\phi_{\mathrm{m}}=\frac{2 \pi L R}{\lambda} \frac{\mathrm{d} n}{\mathrm{~d} N} \frac{\mathrm{d} N}{\mathrm{~d} I} V_{0} \cos \left(\omega_{\mathrm{m}} t\right)=-64.9 V_{0} \cos \left(\omega_{\mathrm{m}} t\right)
$$

If the SOA is replaced by a $\mathrm{LiNbO}_{3}$ modulator, the index change induced by an external electric field $E(t)$ can be given as

$$
\Delta n(t)=\frac{r_{a b} n_{0}^{3}}{2} E(t)
$$

where $r_{a b}$ is the appropriate electrooptic coefficient with a value of about $1.88 \times 10^{-11} \mathrm{~m} / \mathrm{V}$ (calculated based on an electric field of $106 \mathrm{~V} / \mathrm{m}$ applied to a crystal of $\mathrm{LiNbO}_{3}$ producing a fractional index change of about $0.01 \%$ ) and $n_{0}=2.2$ is the refraction index without bias (based on $n_{\mathrm{o}}=2.23, n_{e}=2.15$ ) [14]. With a sinusoidal modulation signal $V(t)=V_{0} \cos \left(\omega_{\mathrm{m}} t\right)$ applied, the phase change at the wavelength $\lambda$ can be expressed as

$$
\Delta \phi_{\mathrm{m} \_\mathrm{Li}}(t)=\frac{\pi r_{a b} n_{0}^{3} l}{\lambda} \frac{V_{0}}{d} \cos \left(\omega_{\mathrm{m}} t\right) .
$$

If the crystal length is $l=300 \mu \mathrm{m}$, the electrode separation is $d=2 \mu \mathrm{m}$, and the voltage applied to the crystal is $V_{0}$, the phase change that resulted from the external modulation signal is

$$
\Delta \phi_{\mathrm{m} \_\mathrm{Li}}=6.08 \times 10^{-2} V_{0} \cos \left(\omega_{\mathrm{m}} t\right) .
$$

Compared with (11), the phase-shift effect introduced by the high-speed $\mathrm{LiNbO}_{3}$ phase modulator is much weaker than that by the SOA at low frequency ( $\sim$ in kilohertz). Therefore, to obtain the same phase shift, the required voltage by a $\mathrm{LiNbO}_{3}$ phase modulator is much higher than that by an SOA.

\section{EXPERIMENT AND RESULT}

The configuration of the proposed multiwavelength EDF laser is shown in Fig. 2. In the laser cavity, a 20-m EDF pumped by a $980-n m$ pump laser through a $980-n m$ pump coupler is used as the gain medium. The power of the 980-nm pump laser is about $200 \mathrm{~mW}$ with a pumping current of $250 \mathrm{~mA}$. An SOA driven by a sinusoidal signal is employed as a phase modulator to suppress the homogeneous line broadening. Note that the SOA is biased below the transparent point; no gain is obtained from the SOA. The input light polarization state to the SOA is adjusted by a polarization controller (PC1). The unidirectional operation of the laser is ensured by the isolator and the circulator. The circulator and the chirped fiber Bragg grating (CFBG) act as a bandpass filter. The Lyot-Sagnac loop is composed of a 3-dB coupler, a PC, and two sections of high-birefringent (HiBi) fibers serving as a wavelength selector. The PC2 is used to adjust the polarization state of the Lyot-Sagnac loop. The output of the laser is monitored by an optical spectrum analyzer (OSA) through a 10:90 optical coupler (OC1).

In principle, the input light to the $3-\mathrm{dB}$ coupler (OC2) is split into two counterpropagating waves. Each wave is decomposed into $2^{2}$ waves after traveling though the HiBi fibers. Depending on the phase difference, birefringence, length of fibers, and polarization state of the PC, the interference pattern could be obtained at the output port of the Lyot-Sagnac filter. The 
transmission function of a Lyot-Sagnac loop filter can be easily derived using Jones matrices and can be written as [15]

$$
\begin{aligned}
T\left(\theta_{1}, \theta_{2}\right)=[ & \sin \left(\frac{\pi}{\lambda} \Delta n\left(L_{1}+L_{2}\right)\right) \sin \left(\frac{\theta_{1}+\theta_{2}}{2}\right) \\
& \times \cos \left(\frac{\theta_{1}-\theta_{2}}{2}\right)+\sin \left(\frac{\pi}{\lambda} \Delta n\left(L_{1}-L_{2}\right)\right) \\
& \left.\times \cos \left(\frac{\theta_{1}+\theta_{2}}{2}\right) \sin \left(\frac{\theta_{1}-\theta_{2}}{2}\right)\right]^{2}
\end{aligned}
$$

where $\Delta n$ is the fiber birefringence, $\lambda$ is the wavelength, $L_{1}$ and $\theta_{1}$ are the length and the polarization rotation angle of the first segment fiber, and $L_{2}$ and $\theta_{2}$ are the length and the polarization rotation angle of the second segment fiber. In the experiment, the HiBi fibers are Corning HiBi fiber with $B=4.0 \times 10^{-4}$, $L_{1}=8.75 \mathrm{~m}$, and $L_{2}=22.25 \mathrm{~m}$. By adjusting the polarization state, the wavelength spacing could be tuned. Note that instead of using a Lyot-Sagnac loop, a sampled fiber Bragg grating (SFBG) can also be used for wavelength selection. In our experiment, an SFBG with a wavelength spacing of $0.5 \mathrm{~nm}$ is used to achieve multiwavelength lasing.

Fig. 3(a) shows the transmission spectrum of the LyotSagnac loop. The spectrum is measured using an OSA with an amplified spontaneous emission (ASE) broadband source applied to the input of the loop. It can be seen that the loop can be considered as a band-unlimited comb filter with a wavelength spacing of $0.39 \mathrm{~nm}$. The total insertion loss of the loop is $7.5 \mathrm{~dB}$.

A CFBG combined with a circulator is used as a bandpass filter to limit the wavelength range of the band-unlimited LyotSagnac loop filter. The CFBG has a linearly increased period from 537.71 to $540.35 \mathrm{~nm}$ with a full-width at half-maximum of $7.8 \mathrm{~nm}$. The central wavelength of the CFBG is $1559.9 \mathrm{~nm}$. The total loss of the CFBG and the circulator is $4 \mathrm{~dB}$ at $1559.9 \mathrm{~nm}$

The APSOA1015D SOA made by Axon Photonics was used in the experiment. The SOA is a GaInAs-buried heterostructure commercial amplifier with a cavity length of 500-600 $\mu \mathrm{m}$. In order to apply a modulation signal to the SOA to achieve the refractive-index modulation, the dc bias and the sinusoidal signal from a function generator are applied to the SOA via a bias tee. The SOA exhibits a transparent current of $\sim 110 \mathrm{~mA}$ and a $3-\mathrm{dB}$ bandwidth of $75 \mathrm{~nm}$ around $1550 \mathrm{~nm}$. Its peak smallsignal gain over the bandwidth is $15 \mathrm{~dB}$ with a polarizationdependent gain of $0.5 \mathrm{~dB}$. The noise figure of the $\mathrm{SOA}$ is $9 \mathrm{~dB}$. With an 86-mA bias current, the SOA has about $2 \mathrm{~dB}$ of absorption loss. To evaluate the performance of the homogeneous line broadening suppression, we focus on two parameters of the multiwavelength laser: the simultaneous lasing wavelength number and the minimum wavelength spacing. The stronger the homogeneous line broadening, the less the lasing wavelength number and the wider the wavelength spacing. Fig. 4(a) shows the lasing spectrum of the fiber laser with the SOA biased below the transparent point at $86 \mathrm{~mA}$ but without a sinusoidal modulation signal applied. It can be seen that very few lasing wavelengths can be generated because of the strong EDF homogeneous line broadening. By carefully adjusting the

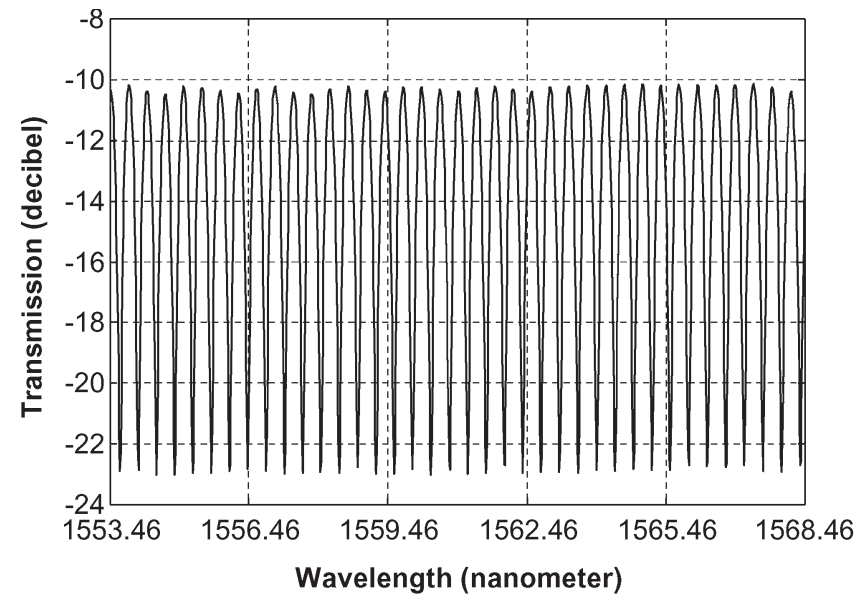

(a)

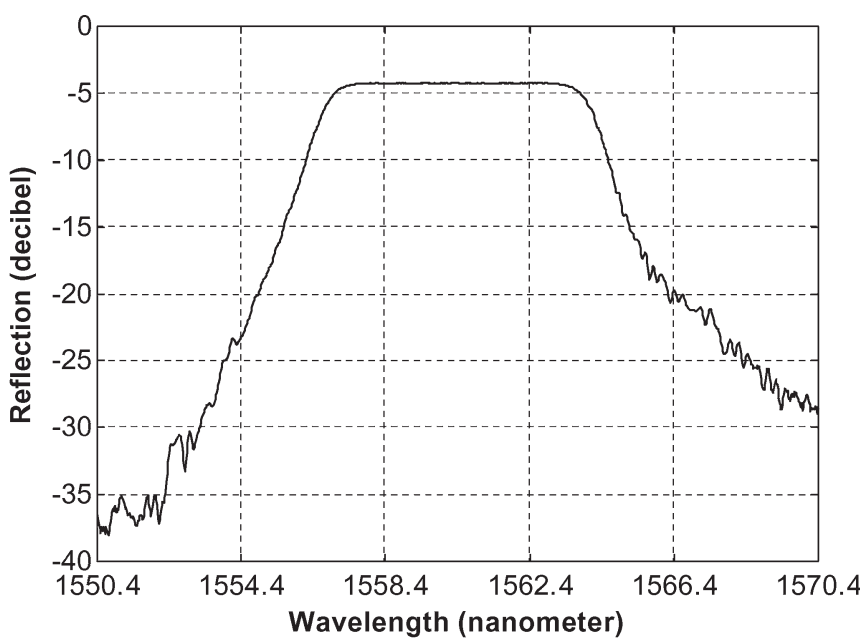

(b)

Fig. 3. (a) Transmission spectrum of the Lyot-Sagnac loop filter. (b) Reflection spectrum of the CFBG.

polarization state (PC1), a stable lasing is achieved with a maximum lasing number of two and a wavelength spacing greater than $1.5 \mathrm{~nm}$. If the fiber laser has a lasing wavelength number more than three or the wavelength spacing is less than $1.5 \mathrm{~nm}$, the lasing becomes unstable and the power fluctuation occurs at all of the lasing wavelengths. If the wavelength spacing is further decreased to less than $0.5 \mathrm{~nm}$, only one wavelength can survive. The same results are obtained if no SOA is incorporated into the laser. The results indicate that incorporating an SOA without gain is not helpful for the suppression of the homogeneous line broadening induced by the EDF, although the SOA is an inhomogeneous medium. The reason is that when the SOA is biased below the transparent point, it simply acts as a linear passive component instead of a gain medium. The lasing modes will still compete with each other in the cavity due to the strong homogeneous line broadening of the EDF, and hence only a single wavelength can be dominant.

By applying a $30-\mathrm{kHz}$ sinusoidal signal to the SOA with an $80-\mathrm{mA}$ bias current, homogeneous line broadening is effectively suppressed and a stable multiwavelength lasing is generated. The output spectrum is shown in Fig. 4(b). It can be 


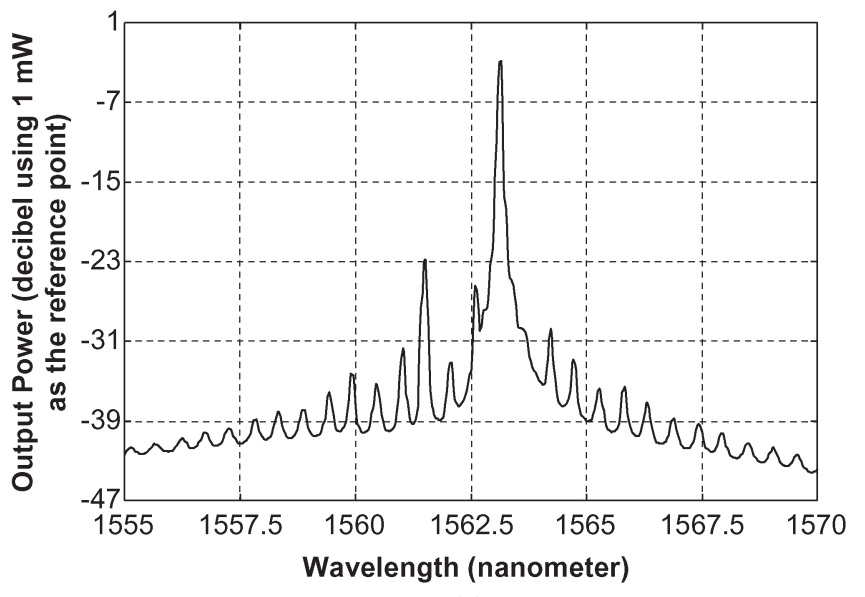

(a)

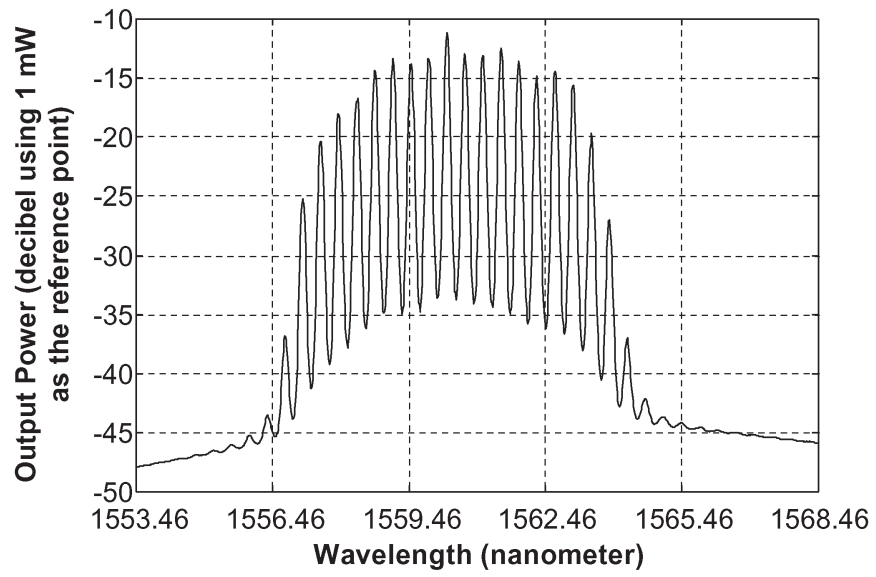

(b)

Fig. 4. Spectra of the fiber laser when the Lyot-Sagnac loop is employed as the wavelength selection filter. (a) SOA biased at $86 \mathrm{~mA}$ without modulation and (b) with the SOA modulated at $30 \mathrm{kHz}$.

seen that 16 stable wavelengths are generated simultaneously. The total output power is $6.3 \mathrm{dBm}$, which can be further increased if an additional EDF is incorporated into the laser cavity to compensate for the insertion loss caused by the passive component [6]. The 3-dB bandwidth is measured to be $0.05 \mathrm{~nm}$ with an identical wavelength spacing of $0.39 \mathrm{~nm}$. The lasing wavelengths can be changed by adjusting the PC2 in the laser cavity to meet the particular requirement.

To further investigate the performance of the fiber laser at smaller wavelength spacing, the Lyot-Sagnac loop comb filter is tuned to have a wavelength spacing of $0.19 \mathrm{~nm}$. Fig. 5 shows the output spectrum at room temperature. A maximum of 26 wavelengths with wavelength spacing of $0.19 \mathrm{~nm}$ are generated.

\section{Discussion}

The effects of the SOA bias current, modulation frequency, modulation voltage, and modulation wave type on the performance of the multiwavelength laser are investigated. During the experiment, when the SOA is driven by a constant current below the transparent point $(\sim 110 \mathrm{~mA})$, the SOA acts as a passive component with certain absorption determined by the

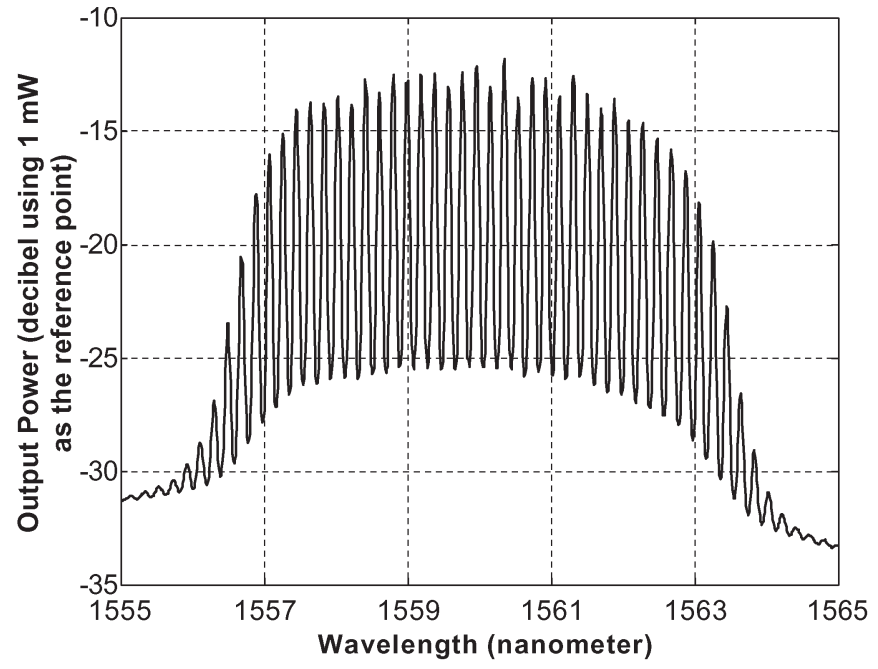

Fig. 5. Spectrum of the fiber laser when the wavelength spacing of the Lyot-Sagnac loop comb filter is tuned to $0.19 \mathrm{~nm}$.

bias current. With a bias current lower than $40 \mathrm{~mA}$, the lasing cannot be obtained because the high absorption of the SOA results in a net gain below the threshold in the laser cavity. With a dc-bias current from 40 to $100 \mathrm{~mA}$, the multiwavelength lasing can be achieved by applying a low-frequency sinusoidal modulation signal ranging from 20 to $300 \mathrm{kHz}$. Since the index change in the SOA is around several tens of microseconds, which is much shorter than the erbium ion response time ( $\sim$ in milliseconds), none of the lasing wavelengths can obtain the competition merit over others by the erbium ion homogeneous line broadening. It also verifies that the modulation applied to the SOA is not performing a mode-locking operation since the modulation frequency is not a multiple of the roundtrip frequency of the laser cavity. A different modulation frequency affects the amount of frequency shift of the SOA. It is found that to maintain the same signal-to-noise ratio (SNR), for a higher modulation frequency, a higher modulation voltage is required. For instance, to achieve an SNR of $20 \mathrm{~dB}$, the modulation voltage for a modulation frequency of $20 \mathrm{kHz}$ is $10 \mathrm{mV}$, while it is $622 \mathrm{mV}$ for a modulation frequency of $200 \mathrm{kHz}$. The results coincide with our theoretical analysis in Section II that a higher modulation voltage is required for a higher modulation frequency to maintain the same frequency shift. Fig. 6 shows the relationship between the required modulation voltage and the modulation frequency.

Moreover, it is found that multiwavelength lasing is achieved by applying not only a sinusoidal wave or a saw-tooth but also a square wave, which is impossible in the fiber laser using a phase modulator [7]. This proves our theoretical analysis that use of an SOA is more effective in the suppression of the homogeneous line broadening than a phase modulator. To further confirm the above conclusion, the SOA in the laser cavity is replaced by a $10-\mathrm{GHz} \mathrm{JDSU} \mathrm{LiNbO}_{3}$ phase modulator. It is found that only a single wavelength can be generated. If the Lyot-Sagnac filter is replaced by the SFBG with a wider wavelength spacing of $0.5 \mathrm{~nm}$, a three-wavelength lasing is obtained, as shown in Fig. 7. In the experiment, the phase modulator is driven by a $12-\mathrm{V}$ sinusoidal signal with a modulation frequency 


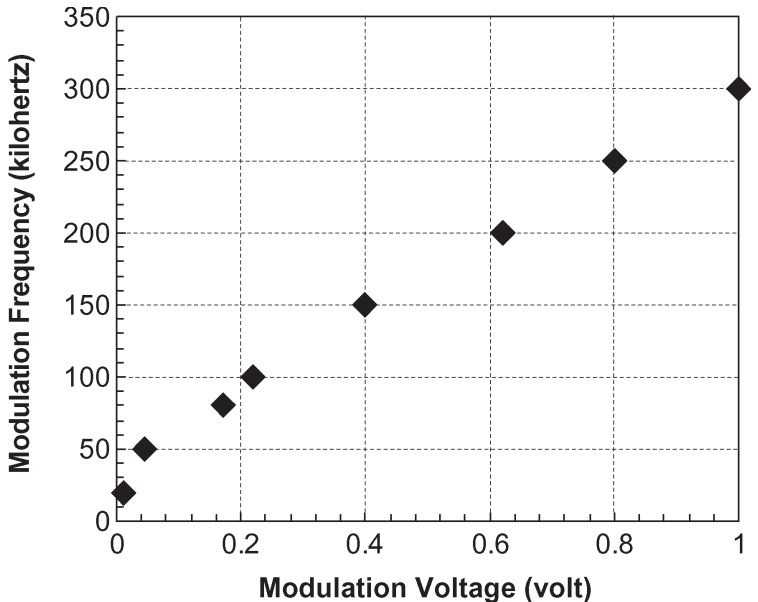

Fig. 6. Modulation frequency versus the modulation voltage of the SOA to achieve the same SNR.

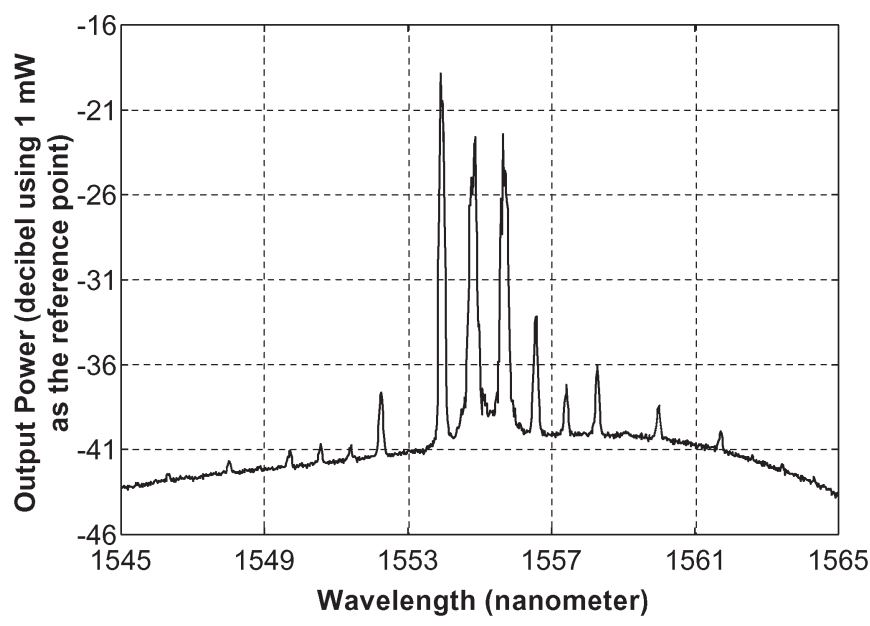

Fig. 7. Output spectrum of the fiber laser with a $\mathrm{LiNbO}_{3}$ phase modulator incorporated. The wavelength selection is performed by the SFBG.

of $25-30 \mathrm{kHz}$. Note that the modulation voltage is much higher than that for the SOA (several tens of millivolts), and the modulation frequency range is also much narrower than that for the SOA $(20-300 \mathrm{kHz})$. Also, the stability of the proposed fiber laser is investigated during the experiment. The output optical spectra taken at a 10-min interval are recorded and compared; no significant variations in amplitudes of the multiwavelengths are observed [9]. However, for the fiber laser using a $\mathrm{LiNbO}_{3}$ modulator, the peak power at all lasing wavelengths fluctuates all the time with a maximum variation of more than $5 \mathrm{~dB}$ and the maximum wavelength number is only five compared with 26 for the SOA-based fiber laser. The results indicate that the stability of the fiber laser using a $\mathrm{LiNbO}_{3}$ phase modulator is much poorer than that with an SOA-based phase modulator, which further confirms that the frequency shift introduced by the SOA is more significant than that by the $\mathrm{LiNbO}_{3}$ phase modulator; better homogeneous line broadening suppression is obtained.

Note that multiwavelength lasing at room temperature can also be implemented by using an all-fiber phase modulator [7]. An all-fiber phase modulator is usually built based on a PZT by wrapping several meters of single-mode fiber around the PZT.
Stable multiwavelength operation can be realized by applying a high-voltage sinusoidal signal to the PZT, with the periodic phase shift resulting from the rapid stretching of the fiber. There are two major problems related to the use of such an all-fiber phase modulator. First, it is very bulky. Second, the fiber laser cavity length is much longer than that using an SOA-based phase modulator since the wrapped fiber has a length of several meters. In addition, the extra tension applied to the fiber would also reduce its lifetime.

\section{CONCLUSION}

A theoretical and experimental study of a multiwavelength fiber laser using an semiconductor optical amplifier (SOA) as a phase modulator to suppress the homogeneous line broadening was presented. Theoretical analysis showed that the SOAbased phase modulator was more effective than the $\mathrm{LiNbO}_{3}$ phase modulator in producing phase or frequency shift for the fiber laser and hence leading to an improved homogeneous line broadening suppression. Stable lasing with multiple wavelengths up to 26 and wavelength spacing as small as $0.19 \mathrm{~nm}$ was demonstrated at room temperature when the SOA was biased below the transparent point with a low-voltage sinusoidal signal applied. The effects of modulation voltage, frequency, and modulation signal type on the performance of the multiwavelength laser were investigated.

\section{REFERENCES}

[1] S. Yamashita and K. Hotate, "Multiwavelength erbium-doped fiber laser using intracavity etalon and cooled by liquid nitrogen," Electron. Lett., vol. 32, no. 14, pp. 1298-1299, 1996.

[2] N. Park and P. F. Wysocki, "24-line multiwavelength operation of erbiumdoped fiber-ring laser," IEEE Photon. Technol. Lett., vol. 8, no. 11, pp. 1459-1461, Nov. 1996.

[3] J. Yao, J. P. Yao, Y. Wang, S. C. Tjin, Y. Zhou, Y. L. Lam, J. Liu, and C. Lu, "Active mode locking of multi-wavelength fiber ring laser," Opt. Commun., vol. 191, no. 3-6, pp. 341-345, May 2001.

[4] O. Graydon, W. H. Loh, R. I. Laming, and L. Dong, "Triple-frequency operation of an Er-doped twincore fiber loop laser," IEEE Photon. Technol. Lett., vol. 8, no. 1, pp. 63-65, Jan. 1996.

[5] Y. Zhao, C. Shu, S. P. Li, H. Ding, and K. T. Chan, "Multiple wavelength operation of a unidirectional Er-doped fiber ring laser with optical feedback," in Tech. Dig. Conf. Laser and Electro-optics (CLEO), Baltimore, MD, 1997, p. 396, CThL65.

[6] A. Bellemare, M. Karasek, M. Rochette, S. Larochelle, and M. Tetu, "Room temperature multifrequency erbium-doped fiber lasers anchored on the ITU frequency grid," J. Lightw. Technol., vol. 18, no. 6, pp. 825831, Jun. 2000.

[7] K. Zhou, D. Zhou, F. Dong, and N. Q. Ngo, "Room-temperature multiwavelength erbium-doped fiber ring laser employing sinusoidal phasemodulation feedback," Opt. Lett., vol. 28, no. 11, pp. 893-895, Jun. 2003.

[8] J. Yao, J. P. Yao, and Z. Deng, "Multiwavelength actively mode-locked fiber ring laser with suppressed homogeneous line broadening and reduced supermode noise," Opt. Express, vol. 12, no. 19, pp. 4529-4534, Sep. 2004.

[9] J. Yao, J. P. Yao, Z. Deng, and J. Liu, "Multiwavelength erbium-doped fiber ring laser incorporating an SOA-based phase modulator," IEEE Photon. Technol. Lett., vol. 17, no. 4, pp. 756-758, Apr. 2005.

[10] A. E. Siegman, Lasers. Mill Valley, CA: Univ. Sci., 1986.

[11] R. Slavk and S. LaRochelle, "Frequency shift in a fiber laser resonator," Opt. Lett., vol. 27, no. 1, pp. 28-30, Jan. 2002.

[12] L. Gillner, "Modulation properties of a near traveling-wave semiconductor laser amplifier," Proc. Inst. Elect. Eng. Optoelectron., vol. 139, no. 5, pp. 331-338, Oct. 1992.

[13] J. Mellis, "Direct optical phase modulation in semiconductor laser amplifier," Electron. Lett., vol. 25, no. 10, pp. 679-680, May 1989. 
[14] Practical Uses and Applications of Electro-Optic Modulators, Application notes. San Jose, CA: New Focus Inc. [Online]. Available: http:// www.newfocus.com/Online_Catalog/Literature/apnote2.pdf

[15] C. Kim, Y. Han, R. M. Sova, U. Paek, Y. Chung, and J. U. Kang, "Optical fiber modal birefringence measurement based on Lyot-Sagnac interferometer," IEEE Photon. Technol. Lett., vol. 15, no. 2, pp. 269-271, Feb. 2003.

Jian Yao received the M.Eng. degree in electrical and electronic engineering from Nanyang Technological University, Singapore, in 2001.

She was a Senior Engineer at Wuhan Research Institute of Posts and Telecom (WRI), Wuhan, China; Trillium Photonics Inc., Ottawa, ON, Canada; and Intelligent Photonic Control, Ottawa, ON, Canada, working on photonic product development. She also worked as a Research Associate at the University of Ottawa, Ottawa, ON, Canada, where she focused on the research and development of fiber lasers. She is currently a Senior Engineer at Oplink Inc., San Jose, CA. Her current interests are fiber lasers and next-generation networks.

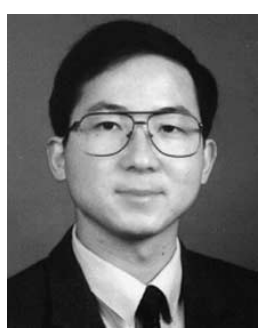

Jianping Yao (M'99-SM'01) received the Ph.D. degree in electrical engineering from the Université de Toulon, France, in 1997.

From January 1998 to July 1999 , he was a Research Fellow, and from July 1999 to December 2001, was an Assistant Professor, both at the School of Electrical and Electronic Engineering, Nanyang Technological University, Singapore. He was an Invited Professor at the Institut National Polytechnique de Grenoble, France, from January to March 2005. $\mathrm{He}$ is now an Associate Professor at the School of Information Technology and Engineering, University of Ottawa, Ottawa, ON, Canada. He has published over 100 papers in refereed journals and conference proceedings. His current research interests include optical signal processing, optically controlled phased array antennas, photonic generation of microwave signals, radio-over-fiber systems, fiber lasers and amplifiers, broadband infrared wireless home networking, and fiber-optic sensors.

Dr. Yao is a Member of The International Society for Optical Engineers (SPIE) and the Optical Society of America (OSA).

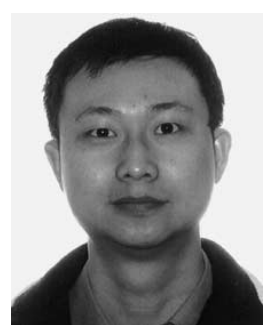

Zhichao Deng received the B.Eng. degree in electrical engineering from Xidian University, China, in 1993. He is currently working toward the M.A.Sc. degree in electrical engineering at the University of Ottawa, Ottawa, ON, Canada

He was an Engineer at China Telecom, Beijing, China, working on satellite and mobile communication. His current research interests include fiber lasers, mode-locked lasers, and microwave photonics.
Jian Liu received the Ph.D. degree in electrical and electronic engineering from Nanyang Technological University, Singapore, in 2002.

He was an Engineer/Researcher at Wuhan Research Institute of Post and Telecom (WRI), Wuhan, China; JDS Uniphase, Ottawa, ON, Canada; and University of Ottawa, Ottawa, ON, Canada; all focusing on design and measurement of optoelectronic devices and systems. He is currently a Natural Sciences and Engineering Research Council of Canada (NSERC) Postdoctoral Fellow at the Institute for National Measurement Standards, National Research Council of Canada. His current interests include the development of high precision standard measurement instruments and material (pearlescent coatings, semiconductor thin films, diffractive gratings, and nanowires) characterization metrology. 